Research Paper

\title{
ERG Tumor Type is Less Frequent in High Grade and High Stage Prostate Cancers of Chinese Men
}

\author{
Jiang Baohong ${ }^{\circledR}$, Jason Sedarsky², Shiv Srivastava², Isabell Sesterhenn ${ }^{3}$, Albert Dobi², Li Quanlin ${ }^{\circledR}$ \\ 1. Department of Urology, The First Affiliated Hospital of Dalian Medical University, Dalian, Liaoning, China \\ 2. Department of Surgery, Uniformed Services University of the Health Sciences and the Walter Reed National Military Medical Center, Bethesda, Maryland, \\ USA \\ 3. The Joint Pathology Center, Silver Spring, Maryland USA
}

$\triangle$ Corresponding authors: Li Quanlin (Phone: (86)-13604942808 AND E-mail: liq2808@163.com) and Jiang Baohong (Phone: (86)-15898640590 AND E-mail: jiangbaohongcn@163.com). Department of Urology, The First Affiliated Hospital of Dalian Medical University, No. 222 Zhongshan Road, Dalian 116011, Liaoning, China.

(c) Ivyspring International Publisher. This is an open access article distributed under the terms of the Creative Commons Attribution (CC BY-NC) license (https://creativecommons.org/licenses/by-nc/4.0/). See http://ivyspring.com/terms for full terms and conditions.

Received: 2018.09.17; Accepted: 2019.01.31; Published: 2019.05.12

\begin{abstract}
Background: The incidence of prostatic adenocarcinoma has been rapidly increasing among Chinese men. This alarming trend prompted evaluations of early causal genomic alterations known to drive prostate tumorigenesis. Recurrent activation of the ETS-Related Gene (ERG) by genomic rearrangements is the most recognized early event in prostate cancer. Following the initial detection of ERG rearrangement at gene expression and genomic and levels, development of diagnostic quality antibodies against ERG oncoprotein have streamlined the rapid assessment of ERG frequencies world-wide. Unexpectedly, these studies revealed highest frequencies of ERG among Caucasian descents, lower frequencies among African Americans and even lower prevalence of ERG among Asian men.

Objective: To asses in a prospective study ERG frequencies, clinico-pathological and prognostic associations of ERG among prostate cancer patients of the Dalian region of Northeast China, by an established immunohistochemical procedure that have been used in studies world-wide.

Methods: Formalin fixed paraffin embedded specimens donated by patients $(\mathrm{N}=50)$ diagnosed with prostatic adenocarcinoma who underwent transurethral resection of the prostate (TURP) between 2007 and 2012 were evaluated for ERG by immunohistochemistry.

Results: Of the 50 cases, $13 / 50$ (26.0\%) tumors were positive for ERG. In all cases, normal prostatic epithelial were ERG negative. ERG was more frequently detected in the lower Gleason score $(\leq 7)$ and low T-stage. Consistent with reports from Asian countries the results of our study shows lower overall frequencies of ERG positive tumors when compared to reports from Western countries.

Conclusion: The intriguing association of even lower ERG frequencies with high Gleason scores and higher T-stages provides impetus for current driver gene discoveries focused on the predominantly ERG negative prostate cancers of Asian men.
\end{abstract}

Key words: prostate cancer; ERG; oncogene; Chinese; high-grade tumors

\section{Introduction}

In Western countries the incidence of prostate cancer $(\mathrm{CaP})$ remained constantly high [1]. In contrast, there has been a rapid increase in prostate cancer incidence in China for reasons that remain to be understood [2]. In the context the unprecedented global racial/ethnic dynamics, better understanding of the rapid increases of $\mathrm{CaP}$ incidence in China will likely have broader implications. Discoveries and characterization of $\mathrm{CaP}$ driver gene alterations have been providing new opportunities for risk stratification and future targeted therapies [3-8].

Among these finding the recurrent activation 
and overexpression of the V-Ets Avian Erythroblastosis Virus E26 Oncogene Homolog, ERG oncogene, has been best characterized and understood [8,9]. Although, the detection of ERG activation has been streamlined at genomic levels by fluorescence in situ hybridization (FISH) assay [10] and at mRNA expression levels [9]. However, immunohistochemical (IHC) detection of ERG that is more compatible with routine pathology settings was challenging in the absence of specific anti-ERG antibodies [11]. Development of the first highly specific antibodies has led to the rapid evaluation of ERG prevalence in $\mathrm{CaP}[12,13]$. Comparative assessments of ERG rearrangements at genomic and protein levels revealed close agreement between FISH and the two introduced IHC methodologies [14]. Along these lines, world-wide assessments of ERG in different ethnic groups at various geographic regions highlighted unexpected findings. Highest frequency of ERG rearrangement and ERG oncoprotein expression has been reported among CaP patients of Caucasian descent (50-60\%), whereas, among African Americans ERG frequency was significantly lower (28\%) [15-19]. Remarkably, the ERG frequency was even lower in high grade tumors of African Americans men (16\%) and in CaPs of African men (18\%) [20,21]. Lower frequency of ERG among Asian patients has been reported at genomic, gene expression or protein levels [22]. These findings have led to new insights into the CaP genomes of ERG negative tumor types of Asian men [23,24]. Although the examined $\mathrm{CaP}$ specimens in world-wide ERG frequency studies included biopsy or TURP or tissue microarray (TMA) or whole mounted radical prostatectomy specimens and were assayed by either reverse transcription-polymerase chain reaction (RT-PCR), FISH and/or IHC, the overall conclusion is that ERG is most prevalent among CaP patients of Western countries.

In the current study we have evaluated ERG frequencies of prostate cancers from the Northeastern region of China that has not been examined before evaluating TURP specimens $(\mathrm{N}=50)$ by IHC and assessed the association of ERG oncoprotein detection with Gleason grade, Gleason score and T-stages.

\section{Materials and Methods}

\section{Patient cohort}

Our study was approved by the ethical committee of the First Affiliated Hospital of Dalian Medical University. All participants signed informed consent before enrolment. Formalin fixed paraffin embedded specimens $(\mathrm{N}=50)$ donated by 50 patients diagnosed with prostatic adenocarcinoma who underwent transurethral resection of the prostate
(TURP) as the primary treatment at Department of Urology, the First Affiliated Hospital of Dalian Medical University between 2007 and 2012 were evaluated for ERG protein expression by immunohistochemistry (IHC). None of the patients received prior hormone ablation or radiation treatments. Serum for PSA measurement was collected prior to digital rectal examination (DRE), transrectal ultrasonography (TRUS) or cystoscopy to avoid procedures that may affect PSA levels. Complete data fields of PSA, type-B ultrasonic, X-ray, CT/MRI and pathology report were examined for all patients in the study.

\section{Patient selection criteria}

The inclusion criteria for the study included diagnosis with prostatic adenocarcinoma, age between 55 and 85, availability of pre-procedural PSA values of $3 \mathrm{ng} / \mathrm{mL}$ or above, Gleason score 6 or above, clinical stage $\mathrm{T} 1$ or above, eligibility for primary treatment, and TURP procedure performed between 2007 and 2012 in the Department of Urology, the First Affiliated Hospital of Dalian Medical University. Patients who did not meet the inclusion criteria or received prior hormone ablation and/or radiation therapy were excluded from the study.

\section{Immunohistochemistry for ERG oncoprotein detection}

Immunohistochemistry (IHC) was performed to detect the expression of ERG protein in prostate specimens. Representative $4-\mu \mathrm{m}$ cross-sections from each TURP specimen were selected. For IHC procedures the MaxVision staining protocol was followed (Fuzhou Maixin Biotech Co., Ltd.). Paraffin tissue slides were incubated for $12 \mathrm{~h}$ at $65^{\circ} \mathrm{C}$. The sections were deparaffinized by washing twice for 15 min at room temperature in dimethyl benzene. The sections were hydrated in sequential incubation in $100 \%, 100 \%, 95 \%, 95 \%, 90 \%, 80 \%$, and $70 \%$ of ethanol each step for $3 \mathrm{~min}$, and then were equilibrated in $1 \times$ PBS three times, each time for $3 \mathrm{~min}$. For antigen retrieval the sections were incubated in $10 \mathrm{mM}$ EDTA ( $\mathrm{pH}$ 9.0) for $30 \mathrm{~min}$ by high pressure steam boiler followed by slowly cooling down to room temperature. Sections were washed by $1 \times$ PBS three times, each for $3 \mathrm{~min}$. Sections were then blocked in $3 \%$ hydrogen peroxide for $10 \mathrm{~min}$ and were equilibrated by three times $3 \mathrm{~min}$ in $1 \times$ PBS. For the primary antibody reaction sections were incubated with the ERG-MAb mouse monoclonal antibody (9FY, Biocare Medical Inc) at dilution of 1:50 for $60 \mathrm{~min}$ at room temperature. For secondary antibody treatment the sections were incubated with the MaxVision HRP-Polymer anti-Mouse antibody (Fuzhou Maixin 
Biotech Co., Ltd.) for $15 \mathrm{~min}$ and were visualized using a Diaminobenzidine (DAB) detection kit (Beijing Zhongshan-Goldenbridge Biotechnology Co., Ltd.). The sections were counter stained by hematoxylin. Tissue slides were dehydrated sequentially in $85 \%, 95 \%$, and $100 \%$ of ethanol and the dehydration was completed by incubation in xylene. The sections were sealed in neutral gum and examined by microscopy.

\section{ERG immunohistochemical staining assessments, counting and grading}

ERG positive prostate cancer cells showed clear nuclear staining for ERG. Strong ERG staining was detected in endothelial cells which were used as an internal positive control for the IHC procedure. ERG staining was not observed in non-cancerous prostate epithelium. ERG protein expression was microscopically evaluated by two board-certified senior pathologists using double-blind method by nuclear staining intensity and the percentage of ERG positive cancer cells. Each section was examined under high view of microscope (400x), and 100 cells per field were counted. ERG staining was calculated from the staining intensity scores of negative (0) mild $(1+)$, moderate $(2+)$ and marked (3+) multiplied by the percent of positive cells of ERG negative (0), up to $25 \%(1+), 26-50 \%(2+), 51-75 \%(3+), 76-100 \%(4+)$ to determine the total score of 0 negative $(-), 1-4$ weak positive $(+)$, 5-8 moderately positive $(++), 9-12$ strongly positive $(+++)$.

\section{Gleason score and clinical T-stage}

According to the 2005 International Society of Urological Pathology (ISUP) criteria, the prostate adenocarcinoma was divided into five grades, related to the prognosis of patients. The Gleason scores and primary Gleason grades were assessed using pathologic records and slide review by two board-certified pathologists in a double blinded fashion according to the 2002 American Joint Committee on Cancer (AJCC) standards for clinical TNM staging system of prostate adenocarcinoma.

\section{Ultrasound measurement of prostate volume}

The prostate was examined by abdominal ultrasonography in order to determinate whether there were suspicious lesions or nodules in the prostate, and the maximum diameter, transverse diameter and longitudinal diameter were measured respectively. Normal dimensions of the prostate was $4 \mathrm{~cm} \times 3 \mathrm{~cm} \times 2 \mathrm{~cm}$. Prostate volume was calculated using the "Terris formula" (prostate volume V $\left(\mathrm{cm}^{3}\right)=$ anteroposterior diameter $(\mathrm{cm}) \times$ transverse diameter $(\mathrm{cm}) \times$ longitudinal diameter $(\mathrm{cm}) \times 0.52)$.

\section{Statistical Analysis}

For patient characteristics, continuous variables were shown as means and ranges, and categorical variables were presented as frequencies and percentages. Chi -square tests were used to determine the association between the immunohistochemical results of ERG protein and the clinicopathologic variables. The SPSS version 17.0 software (SPSS Inc., Chicago, IL, USA) was used for data analysis. $\mathrm{P}<0.05$ was taken as statistically significant.

\section{Results}

\section{Clinico-pathological characteristics of the study subjects}

The study included 50 patients with a mean age of 69 years (Table 1). The average prostate volume was $51.1 \mathrm{~cm}^{3}$ as measured by abdominal ultrasound. The average of preoperative PSA was $46.6 \mathrm{ng} / \mathrm{ml}$. Gleason score of 5-6 was assigned to $16 \%, 7$ in $22 \%$, and $8-10$ in $62 \%$ of cases. Primary Gleason pattern of $\leq$ 3 was found in $56 \%$ and $4-5$ in $44 \%$ of cases. Clinical stage $\mathrm{T} 1+\mathrm{T} 2$ was seen in $48.0 \%$, and $\mathrm{T} 3+\mathrm{T} 4$ in $52 \%$ of cases.

Table 1. Clinical and pathologic parameters of study subjects $(n=50)$

\begin{tabular}{lll}
\hline Parameters & Mean (median, range) & Number of cases (\%) \\
\hline Age at surgery (year) & $69(76,55-84)$ & \\
$\leq 65$ & & $3(6.0 \%)$ \\
$65-75$ & $24(48.0 \%)$ \\
$>75$ & $23(46.0 \%)$ \\
Prostate volume $\left(\mathbf{c m}^{3}\right)$ & $51.12(10.29-105.65)$ & \\
$\leq 25$ & & $5(10.0 \%)$ \\
$25-50$ & & $31(62.0 \%)$ \\
$>50$ & $14(28.0 \%)$ \\
Preoperative PSA (ng/ml) & $46.56(3.42-100.00)$ & \\
$\leq 4$ & & $3(6.0 \%)$ \\
$4.1-10.0$ & & $7(14.0 \%)$ \\
$10.1-20.0$ & $11(22.0 \%)$ \\
$>20$ & $29(58.0 \%)$ \\
Gleason Score & \\
$5-6$ & $8(16.0 \%)$ \\
7 & & $11(22.0 \%)$ \\
$8-10$ & $31(62.0 \%)$ \\
Primary Gleason pattern & \\
$\leq$ grade 3 & $28(56.0 \%)$ \\
grade 4 or 5 & $22(44.0 \%)$ \\
Clinical T Stage & \\
T1+T2 & $24(48.0 \%)$ \\
T3+T4 & $26(52.0 \%)$ \\
\hline
\end{tabular}

\section{Immunohistochemical detection of ERG protein and the frequency of ERG positive tumors}

Among the examined patients diagnosed with primary prostatic adenocarcinoma, ERG negative tumors in all examined foci were detected in $74 \%$ of 
cases $(n=37)$. ERG positive tumor cells were detected in one or more tumor foci in $26 \%(n=13)$ of cases with predominantly moderate to strong positive nuclear staining (Figure 1). ERG staining was not detected in any prostate gland with normal morphology. Strong and consistent ERG staining was seen in all examined endothelial cells that served as an intrinsic control for the IHC assay.

\section{ERG positive tumor type is more frequent in patients diagnosed with low Gleason score and lower clinical T-stage}

The detection rates of ERG were $47.4 \%$ (9/19) in patients with Gleason score $\leq 7$ in contrast to the $12.9 \%$ (4/31) among patients with Gleason score 8-10 diagnosis indicating a negative correlation between the two groups $(p=0.008)$ (Table 2$)$. Statistical analysis of primary Gleason pattern subgroups, the frequency of detecting ERG positive tumors was significantly different $(p=0.017)$, with ERG positive rate of $39.3 \%$ in primary Gleason pattern $\leq$ grade 3 subgroup in comparison to the grade 4-5 subgroup of only $9.1 \%$ ERG positive tumor detection (Table 2). Among patients with $\mathrm{T} 1+\mathrm{T} 2$ clinical stages the frequency of detecting ERG positive tumors was 41.7\% (10/24). In contrast, only $11.5 \%(3 / 26)$ of patients at $\mathrm{T} 3+\mathrm{T} 4$ clinical stages harbored ERG positive tumors ( $p=$ 0.016). Thus, patients with ERG positive tumors more often fell into the subgroup with lower clinical stages of prostatic adenocarcinoma (Table 3).

\section{Correlation analyses of ERG with age, prostate volume and preoperative PSA ERG and the age of patients}

We examined the correlations of ERG positive and ERG negative tumors in age groups of our study cohort. In $\leq 65,65-75$ years and $>75$ years age groups, ERG expression positive rate were $66.7 \%$ (2 / 3), $16.7 \%$ (4 / 24), and 30.4\% (7 / 23), respectively. The analysis showed no correlation between ERG detection and the age of patients within the examined groups $(p=0.148)$ (Table 4). Also, we assessed correlations between prostate volumes and ERG status. In $\leq 25 \mathrm{~cm}^{3}$ subgroup, $25-50 \mathrm{~cm}^{3}$ subgroup and the $>50 \mathrm{~cm}^{3}$ subgroup, ERG-positive rates were $40 \%$ $(2 / 5), 19.4 \%(6 / 31)$ and $35.7 \%(5 / 14)$, respectively. We found no statistically significant difference in ERG status of tumors along the strata of prostate volumes $(p=0.393)$ (Table 5). To evaluate the correlation of preoperative PSA with the detection of ERG positive tumors we stratified patients based on clinically relevant preoperative PSA values. In the $\leq 4 \mathrm{ng} / \mathrm{ml}$ subgroup, $4.1-10.0 \mathrm{ng} / \mathrm{ml}$ subgroup, $10.1-20.0 \mathrm{ng} / \mathrm{ml}$ subgroup and $>20 \mathrm{ng} / \mathrm{ml}$ subgroup, ERG positive rates were $33.3 \%$ (1 / 3), 28.6\% (2 / 7), 36.4\% (4 / 11), and 20.7\% (6 / 29), respectively. Statistical analysis revealed no correlation between detecting ERG positive tumor type and preoperative PSA levels $(\mathrm{p}=0.771)$ (Table 6).

\section{Discussion}

Activation of the ERG oncogene by genomic fusions is a prostate cancer causing event in CaP [25]. Overexpression of ERG has been noted in over two-third of CaPs of Caucasian descents. Development of ERG-specific antibodies for immunohistochemical detection allowed the rapid race-ethnicity focused world-wide evaluation of ERG [16]. The frequency of ERG is significantly higher in Caucasian, when compared to African American or Asian CaP patients [22]. These findings provide an impetus to explore genomic driver alteration in ERG negative CaPs [26] and discovery of race/ethnicity associated $\mathrm{CaP}$ genomic defects in African Americans $[27,28,29]$ and Asian men [23,24].

Table 2. ERG positive tumor type is detected more frequently among patients diagnosed with lower Gleason score and lower primary Gleason pattern prostatic adenocarcinoma.

\begin{tabular}{lclll}
\hline Gleason score & N & \multicolumn{2}{l}{ ERG protein expression } & p value \\
\cline { 3 - 5 } & & Negative & Positive & \\
\hline $\begin{array}{l}\text { Gleason score } \\
\leq 7\end{array}$ & 19 & $10(52.6 \%, 10 / 19)$ & $9(47.4 \%, 9 / 19)$ & $\underline{\mathbf{0 . 0 0 8}}$ \\
$8-10$ & 31 & $27(87.1 \%, 27 / 31)$ & $4(12.9 \%, 4 / 31)$ & \\
$\begin{array}{l}\text { Primary Gleason pattern } \\
\text { Sgrade 3 }\end{array}$ & & & $\underline{\mathbf{0 . 0 1 7}}$ \\
grade 4 or 5 & 28 & $17(60.7 \%, 17 / 28)$ & $11(39.3 \%, 11 / 28)$ & \\
\hline
\end{tabular}

Table 3. Percentage of detecting ERG positive tumors in clinical T-stage subgroups

\begin{tabular}{lllll}
\hline $\begin{array}{l}\text { Clinical T } \\
\text { stage }\end{array}$ & N & \multicolumn{2}{l}{ ERG status } & p value \\
\cline { 3 - 4 } & & Negative & Positive & \\
\hline T1+T2 & 24 & $14(58.3 \%, 14 / 24)$ & $10(41.7 \%, 10 / 24)$ & $\underline{\mathbf{0 . 0 1 6}}$ \\
T3+T4 & 26 & $23(88.5 \%, 23 / 26)$ & $3(11.5 \%, 3 / 26)$ & \\
\hline
\end{tabular}

Table 4. ERG expression in patients with various age subgroups

\begin{tabular}{lllll}
\hline Age (year) & $\mathbf{N}$ & ERG status & p-value \\
\cline { 3 - 4 } & & Negative & Positive & \\
\hline$\leq 65$ & 3 & $1(33.3 \%, 1 / 3)$ & $2(66.7 \%, 2 / 3)$ & 0.148 \\
$65-75$ & 24 & $20(83.3 \%, 20 / 24)$ & $4(16.7 \%, 4 / 24)$ & \\
$>75$ & 23 & $16(69.6 \%, 16 / 23)$ & $7(30.4 \%, 7 / 23)$ & \\
\hline
\end{tabular}

Table 5. Frequency of detecting ERG positive tumor type in prostate volume subgroups

\begin{tabular}{|c|c|c|c|c|}
\hline \multirow{2}{*}{$\begin{array}{l}\text { Prostate volume } \\
\left(\mathrm{cm}^{3}\right)\end{array}$} & \multirow[t]{2}{*}{$\mathbf{N}$} & \multicolumn{2}{|l|}{ ERG expression } & \multirow[t]{2}{*}{ p-value } \\
\hline & & Negative & Positive & \\
\hline$\leq 25$ & 5 & $3(60.0 \%, 3 / 5)$ & $2(40.0 \%, 2 / 5)$ & 0.393 \\
\hline $25-50$ & 31 & $25(80.6 \%, 25 / 31)$ & $6(19.4 \%, 6 / 31)$ & \\
\hline$>50$ & 14 & $9(64.3 \%, 9 / 14)$ & $5(35.7 \%, 5 / 14)$ & \\
\hline
\end{tabular}


Table 6. Frequency of ERG positive tumors in patients in preoperative PSA subgroups

\begin{tabular}{lllll}
\hline $\begin{array}{l}\text { Preoperative } \\
\text { PSA (ng/ml) }\end{array}$ & N & \multicolumn{2}{l}{ ERG expression } & p-value \\
\cline { 3 - 5 }$\leq 4$ & 3 & $2(66.7 \%, 2 / 3)$ & $1(33.3 \%, 1 / 3)$ & 0.771 \\
$4.1-10.0$ & 7 & $5(71.4 \%, 5 / 7)$ & $2(28.6 \%, 2 / 7)$ & \\
$10.1-20.0$ & 11 & $7(63.6 \%, 7 / 11)$ & $4(36.4 \%, 4 / 11)$ & \\
$>20$ & 29 & $23(79.3 \%, 23 / 29)$ & $6(20.7 \%, 6 / 29)$ & \\
\hline
\end{tabular}

In our study we examined the frequencies of ERG oncoprotein in prostate cancers of Chinese men from the Northeastern region of China. We found an ERG frequency of $26 \%$ in our patient group that is higher than the $10-15 \%$ ERG frequencies reported by similar immunohistochemical methods from other regions of China [30, 31, 32]. We found this regional difference intriguing with the caution of limitations of small sample sizes in our, as well as other similar studies in China on ERG frequencies. The following limitations may contribute to the observed differences between our and other comparable studies. First, the sample size $(\mathrm{N}=50)$ may influenced the observed ERG frequency. Second, the specimens in our study were obtained through TURP procedure as oppose to other studies using biopsy or radical prostatectomy specimens. Third, an independent validation was not included here. However, we have carefully assessed and reviewed independent reports on ERG frequencies from China, including references $[30,31$, 32] and our review [25] highlighting the criteria for ERG immunohistochemical procedures and specimen sampling when independent studies are compared.

In our study, detection of ERG positive tumor type correlated with lower Gleason score $(\leq 7$; $\mathrm{p}=0.008)$, Primary Gleason pattern $(\leq$ grade 3 ; $\mathrm{p}=0.017)$ and lower Clinical T-stage $(\mathrm{T} 1+\mathrm{T} 2 ; \mathrm{p}=0.016)$. These findings are consistent with large cohort studies reporting favorable pathology of CaPs among African American and Caucasian American men [19]. Thus,

\begin{tabular}{|c|c|}
\hline ERG protein expression & Total \\
\hline Negative & $\mathbf{3 7}(\mathbf{7 8 . 0} \%, \mathbf{3 7} / 50)$ \\
\hline Positive & $13(26.0 \%, 13 / 50)$ \\
\hline
\end{tabular}

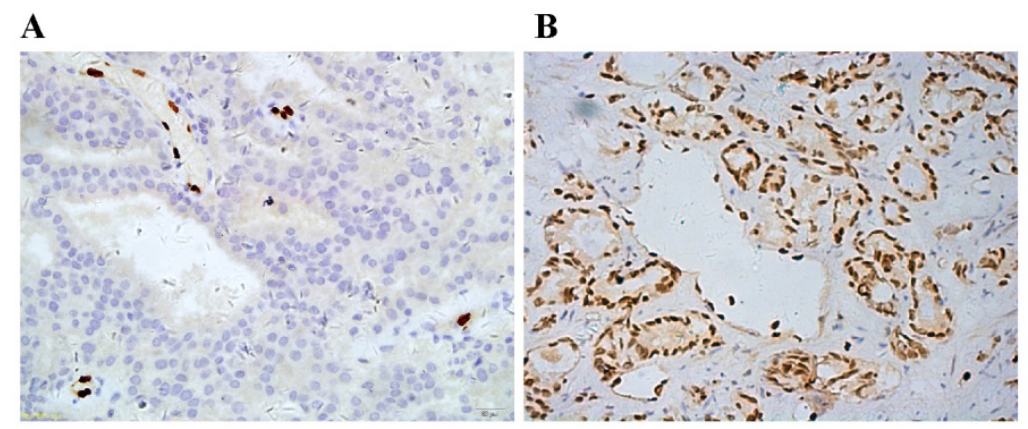

Figure 1. Frequency (upper panel) and representative view fields (lower panels) of ERG negative (A), and ERG positive tumor glands (B) shown in 400X magnification. adverse pathology is more likely associated with patients harboring ERG negative CaP. Age, tumor volume, pre-operative PSA did not show correlation with ERG tumor status in our study.

Typing prostate tumors for driver gene alterations, such as ERG may aid future targeted therapeutic approaches. Current early stage developments of ERG focused therapies target the androgen axis, aim to destabilize ERG mRNA or protein, inhibit ERG interactomes, ERG transcription factor function or cooperating ERG downstream. Indeed, ERG as a transcription factor represents a challenging direct target. However, inhibition of ERG activator kinases or cooperating factors may provide new opportunities for therapeutic interventions $[33,34]$.

Typing of CaPs for ERG is also important in endeavors aiming to discover driver $\mathrm{CaP}$ genomic alteration in ERG negative tumors [26]. Along these lines genome and transcriptome evaluations of $\mathrm{CaPs}$ of Chinese men have revealed high frequency of known, as well as, new genomic alterations. Whole genome sequencing of Chinese men with advanced $\mathrm{CaP}$ indicate higher frequency of TP53 mutations [24]. Remarkably, high frequency of deletions of the CHD1 DNA helicase gene has been reported in $\mathrm{CaP}$ genomes of Chinese men [23]. The deletion of CHD1 may represent a potential target for PARP or platinumbased therapies.

Taken together, typing prostate tumors for ERG and examining correlations of ERG positive or ERG negative tumors with clinico-pathologic features provide important information for further assessment of $\mathrm{CaP}$ driver gene defects among Asian and African men with predominantly ERG negative CaPs.

\section{Acknowledgements}

This research was supported by the National Cancer Institute grant (R01CA162383) to SS; and by USUHS-CPDR fund (HU0001-10-2-0002).

\section{Disclaimer}

The opinions or assertions contained herein are the private ones of the author/speaker and are not to be construed as official or reflecting the views of the Department of Defense, the Uniformed Services University of the Health Sciences or any other agency of the U.S. Government. The identification of specific products, scientific instrumentation, or organization is considered an integral part of the scientific endeavor and does not constitute endorsement or implied endorsement on the part of the author, DoD, or any component agency. 


\section{Competing Interests}

$\mathrm{AD}$ and SS are co-inventors of the CPDR ERG-Mab that has been licensed by Biocare Medical Inc. Other authors declare no conflict of interest.

\section{References}

1. Siegel RL, Miller KD, Jemal A. Cancer statistics, 2018. CA Cancer J Clin. 2018; 68(1):7-30.

2. Chen W, Zheng R, Baade PD, et al. Cancer statistics in China, 2015. CA Cancer J Clin. 2016; 66(2):115-32.

3. Abeshouse A, Ahn J, Akbani R, et al. The Molecular Taxonomy of Primary Prostate Cancer. Cell. 2015; 163(4):1011-25.

4. Robinson DR, Wu YM, Lonigro RJ, et al. Integrative clinical genomics of metastatic cancer. Nature. 2017; 548(7667):297-303.

5. Fraser M, Sabelnykova VY, Yamaguchi TN, et al. Genomic hallmarks of localized, non-indolent prostate cancer. Nature. 2017; 541(7637):359-64.

6. Quigley DA, Dang HX, Zhao SG, et al. Genomic Hallmarks and Structural Variation in Metastatic Prostate Cancer. Cell. 2018; 174(3):758-69.

7. Wedge DC, Gundem G, Mitchell T, et al. Sequencing of prostate cancers identifies new cancer genes, routes of progression and drug targets. Nat Genet. 2018; 50(5):682-92

8. Tomlins SA, Rhodes DR, Perner S, et al. Recurrent fusion of TMPRSS2 and ETS transcription factor genes in prostate cancer. Science. 2005; 310(5748):644-8.

9. Petrovics G, Liu A, Shaheduzzaman S, et al. Frequent overexpression of ETS-related gene-1 (ERG1) in prostate cancer transcriptome. Oncogene. 2005; 24(23):3847-52

10. Perner S, Demichelis F, Beroukhim R, et al. TMPRSS2:ERG fusion-associated deletions provide insight into the heterogeneity of prostate cancer. Cancer Res. 2006; 66(17):8337-41.

11. Rosen P, Sesterhenn IA, Brassell SA, et al. Clinical potential of the ERG oncoprotein in prostate cancer. Nat Rev Urol. 2012; 9(3):131-7.

12. Furusato B, Tan SH, Young D, et al. ERG oncoprotein expression in prostate cancer: clonal progression of ERG-positive tumor cells and potential for ERG-based stratification. Prostate Cancer Prostatic Dis. 2010; 13(3):228-37.

13. Park K, Tomlins SA, Mudaliar KM, et al. Antibody-based detection of ERG rearrangement-positive prostate cancer. Neoplasia. 2010; 12(7):590-8.

14. Braun M, Goltz D Shaikhibrahim Z et al. ERG protein expression and genomic rearrangement status in primary and metastatic prostate cancer--a comparative study of two monoclonal antibodies. Prostate Cancer Prostatic Dis. 2012; 15(2):165-9.

15. Magi-Galluzzi $\mathrm{C}$, Tsusuki $\mathrm{T}$, Elson $\mathrm{P}$, et al. TMPRSS2-ERG gene fusion prevalence and class are significantly different in prostate cancer of Caucasian, African-American and Japanese patients. Prostate. 2011; 71(5):489-97.

16. Rosen P, Pfister D, Young D, et al. Differences in frequency of ERG oncoprotein expression between index tumors of Caucasian and African American patients with prostate cancer. Urology. 2012; 80(4):749-53.

17. Khani F, Mosquera JM, Park K, et al. Evidence for molecular differences in prostate cancer between African American and Caucasian men. Clin Cancer Res. 2014; 20(18):4925-34

18. Blattner M, Lee DJ, O'reilly $\mathrm{C}$, et al. SPOP mutations in prostate cancer across demographically diverse patient cohorts. Neoplasia. 2014; 16(1):14-20.

19. Cullen J, Young D, Chen Y, et al. Predicting Prostate Cancer Progression as a Function of ETS-related Gene Status, Race, and Obesity in a Longitudinal Patient Cohort. Eur Urol Focus. 2018; 4(6):818-24.

20. Farrell J, Young D, Chen Y, et al. Predominance of ERG-negative high-grade prostate cancers in African American men. Mol Clin Oncol. 2014; 2(6):982-6.

21. Zhou CK, Stanczyk FZ, Hafi M, et al. Circulating and intraprostatic sex steroid hormonal profiles in relation to male pattern baldness and chest hair density among men diagnosed with localized prostate cancers. Prostate. 2017; 77(16):1573-82

22. Sedarsky J, Degon M, Srivastava S, Dobi A. Ethnicity and ERG frequency in prostate cancer. Nat Rev Urol. 2018; 15(2):125-31.

23. Ren S, Wei GH, Liu D, et al. Whole-genome and Transcriptome Sequencing of Prostate Cancer Identify New Genetic Alterations Driving Disease Progression. Eur Urol. doi: 10.1016/j.eururo.2017.08.027.

24. Su F, Zhang W, Zhang D, et al. Spatial Intratumor Genomic Heterogeneity within Localized Prostate Cancer Revealed by Single-nucleus Sequencing. Eur Urol. 2018; 74(5):551-9.

25. Rubin MA, Maher CA, Chinnaiyan AM. Common gene rearrangements in prostate cancer. J Clin Oncol. 2011; 29(27):3659-68.

26. Xiao $Q$, Sun $Y$, Dobi $A$, et al. Systematic analysis reveals molecular characteristics of ERG-negative prostate cancer. Sci Rep. 2018; 8(1):12868.

27. Petrovics G, Li H, Stumpel T, et al. A novel genomic alteration of LSAMP associates with aggressive prostate cancer in African American men. EBioMedicine. 2015; 2(12):1957-64

28. Lindquist KJ, Paris PL, Hoffmann TJ, et al Mutational Landscape of Aggressive Prostate Tumors in African American Men. Cancer Res. 2016; 76(7):1860-8.

29. Huang FW, Mosquera JM, Garofalo A, et al. Exome Sequencing of African-American Prostate Cancer Reveals Loss-of-Function ERF Mutations. Cancer Discov. 2017; 7(9):973-83.
30. Jiang $H$, Mao $X$, Huang $X$, et al. TMPRSS2.ERG fusion gene occurs less frequently in Chinese patients with prostate cancer. Tumour Biol. 2016; 37(9):12397-402.

31. Pan X, Zhang X, Gong J, et al. The expression profile and prognostic value of SPINK1 in initially diagnosed bone metastatic prostate cancer. Prostate. 2016; 76(9):823-33.

32. Xue L, Mao X, Ren G, et al. Chinese and Western prostate cancers show alternate pathogenetic pathways in association with ERG status. Am J Cancer Res. 2012; 2(6):736-44.

33. Mohamed AA, Tan SH, Xavier CP, et al. Synergistic Activity with NOTCH Inhibition and Androgen Ablation in ERG-Positive Prostate Cancer Cells. Mol Cancer Res. 2017; 15(10):1308-17.

34. Mohamed AA, Xavier CP, Sukumar G, et al. Identification of a Small Molecule That Selectively Inhibits ERG-Positive Cancer Cell Growth. Cancer Res. 2018; 78(13):3659-71. 